\title{
Direct measurement of acid permeation into rat oesophagus
}

S Tanaka, S Chu, M Hirokawa, M H Montrose, J D Kaunitz

\begin{abstract}
Background and aims: The early responses of the oesophageal mucosa to acid perfusion may predict subsequent pathology. Mucosal responses to luminal acid may result either from acid permeating through the mucosa or from other unknown transduction mechanisms. In order to better understand the dynamics of acid permeation into the oesophageal mucosa, we measured interstitial $\mathrm{pH}\left(\mathrm{pH}_{\text {int }}\right)$ of the oesophageal basal epithelial layer, pre-epithelial layer thickness, and blood flow in rats in vivo during luminal acid challenge. A novel confocal microscopic technique was used in vitro to measure $\mathrm{pH}_{\text {int }}$ from defined cellular sites in response to luminal and basolateral acidification. Methods: 5-(and-6)-Carboxyfluorescein (CF) and carboxy- seminapthorhodofluor-1 (SNARF-1) fluorescence was used to measure $\mathrm{pH}_{\text {int }}$ by conventional and confocal microscopy, respectively, in urethane anaesthetised rats. Pre-epithelial layer thickness was measured optically with carbon particles as markers. Blood flow was measured with laser Doppler flowmetry. Results: Luminal acidification failed to alter $\mathrm{pH}_{\text {int }}$ in vivo and in vitro, but $\mathrm{pH}_{\text {int }}$ was lowered by modest serosal acidification. Pre-epithelial layer thickness and blood flow increased significantly during luminal surface acid perfusion. Indomethacin had no effect on any acid related response.

Conclusion: In this first dynamic measurement of oesophageal acid permeation and pre-epithelial layer thickness, $\mathrm{pH}_{\text {int }}$ was preserved in spite of high luminal acidity by two complementary techniques. Despite the apparent permeability barrier to acid permeation, oesophageal blood flow and thickness responded to luminal acid perfusion.
\end{abstract}

See end of article for authors' affiliations

Correspondence to: Dr J D Kaunitz, West Los Angeles VA Medical Centre, Bldg 114, Room 217, 11301 Wilshire Blvd, Los Ángeles, CA 90073, USA; jake@ucla.edu 26 November 2002

A cid related oesophageal disorders are currently receiving considerable attention as a consequence of their potentially severe morbidities. Although the late stages of acid related disease, including erosive oesophagitis and metaplastic transformation, are well described, the early responses to acid exposure, which must necessarily precede subsequent pathology, have been subject to lesser number of studies. ${ }^{12}$ Hence a grasp of these early responses could be of use in the prevention of oesophageal damage.

The oesophageal mucosa is highly resistant to refluxed concentrated gastric acid..$^{34}$ Oesophageal resistance to acid is thought to reside in luminal $\mathrm{pH}$ gradients that may result from bicarbonate secretion in some species, resistance to acid permeation due to high resistance intercellular structures, and intrinsic cellular resistance due to acid-base transporters in the basal cell plasma membrane. ${ }^{5}$ As is the case with the gastric and duodenal mucosae, it is likely that unique defence mechanisms are in place that are regulated according to luminal $\mathrm{pH}^{7{ }^{8}}$ Unlike other acid exposed organs, however, the replicating cells within the basal pre-epithelial layer are 25-30 cell layers removed from the luminal surface. ${ }^{9}$ A question thus remains as to how far acid penetrates into the mucosa in the absence of injury. The luminal acid signal is rapidly transduced to submucosal structures, as acute luminal acid exposure produces responses such as changes in ion secretion, blood flow, and prostaglandin production, ${ }^{10-12}$ and chronic exposure to excess luminal acid arguably produces proliferative changes in the basal epithelial layer. ${ }^{13}$ It is not known however whether the signalling for these responses results from submucosal acidification or from alternate mechanisms. The observation that luminal acidification fails to produce much injury to the basal layer, whereas even modest acidification of the serosal surface has a far greater potential for producing cell necrosis, ${ }^{14}{ }^{15}$ is consistent with minimal transmucosal acid permeation. Nevertheless, it is not known if these responses to luminal acid result from actual acid penetration into the basal layer or from indirect effects of luminal acid, such as from neural or other signalling mechanisms, as there are no published studies in which the depth of penetration of luminal acid into the mucosa was measured directly in vivo in the absence of injury.

To address the question of the depth of luminal acid penetration, and also to study the response of two putative mucosal defence mechanisms that may play important roles in the resistance of the stomach and duodenum to acid induced injury, we measured interstitial $\mathrm{pH}\left(\mathrm{pH}_{\mathrm{int}}\right)$, preepithelial layer thickness, and blood flow in the oesophageal mucosa. To accomplish this, we modified techniques used to measure intracellular $\mathrm{pH}\left(\mathrm{pH}_{\mathrm{i}}\right)$, mucus gel thickness, and blood flow in the stomach and duodenum of living rodents ${ }^{1617}$ and a confocal microscopic technique used previously to measure extracellular $\mathrm{pH}^{18}{ }^{19}$ in the stomach and colon. We hypothesised that the oesophagus serves as an effective barrier to penetration of luminal acid, preventing the submucosa from acidifying in the presence of luminal acid.

With in vivo microscopy and confocal in vitro microscopy, we demonstrated for the first time that rat oesophageal $\mathrm{pH}_{\text {int }}$ was maintained despite high luminal acidity. Moreover, we also demonstrated that luminal acid can only superficially penetrate the oesophageal mucosa. Furthermore, our data are consistent with the involvement of mucosal blood flow and a possible participation of the pre-epithelial layer in mucosal protective mechanisms.

\section{MATERIALS AND METHODS} Chemicals

5-(and-6)-Carboxyfluorescein (CF) and carboxyseminapthorhodofluor-1 (SNARF-1) were obtained from

Abbreviations: $\mathrm{pH}_{\text {int }}$ interstitial $\mathrm{pH} ; \mathrm{pH}_{\mathrm{i}}$ intracellular $\mathrm{pH} ; \mathrm{CF}$ 5-(and-6)-carboxy fluorescein; SNARF-1, carboxy-seminapthorhodofluor-1; FI, fluorescent intensity; BGI, background intensity. 


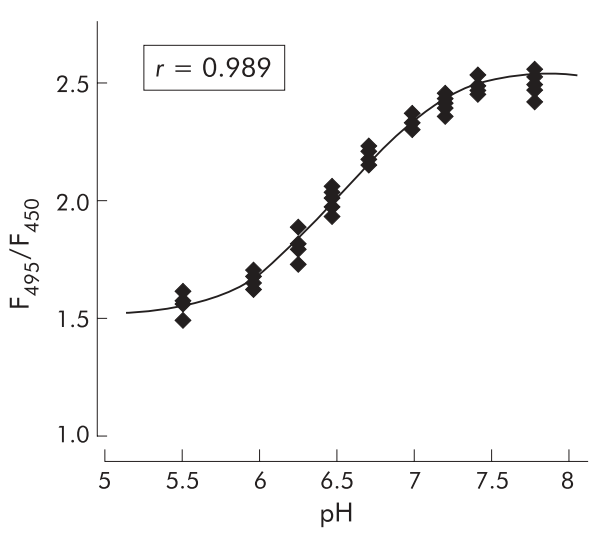

Figure 1 In vitro calibration curve of 5-(and-b-) carboxyfluorescein (CF) fluorescence. The curve was generated by loading excised oesophageal mucosa with $C F$, as described in materials and methods.

Molecular Probes Ltd (Eugene, Ohio, USA). N-2hydroxyethylpiperazine- $N^{\prime}$ - 2-ethanesulphonic acid (HEPES) and other chemicals were obtained from Sigma Chemical Co. (St Louis, Missouri, USA). Krebs solution contains (in $\mathrm{mM}$ ): $136 \mathrm{NaCl}, 2.6 \mathrm{KCl}, 1.8 \mathrm{CaCl}_{2}$, and 10 HEPES at pH 7.0. Krebs solutions were titrated to $\mathrm{pH} 6.5,7.0$, or 7.4 with $1 \mathrm{~N} \mathrm{NaOH}$ $\left(\sim 2-5 \mathrm{mM}\right.$ added $\left.\mathrm{Na}^{+}\right)$, or titrated to $\mathrm{pH} 1.5$ or 1.0 with $5 \mathrm{~N} \mathrm{HCl}$ (15-25 added $\mathrm{Cl}^{-}$). Subsequent to $\mathrm{pH}$ titration, all solutions for in vivo studies were adjusted to 300 mosmol with $\mathrm{NaCl}$. For luminal acid perfusion, Krebs solution was $\mathrm{pH} 1.5$ for the in vitro studies and $\mathrm{pH} 1.0$ for the in vivo studies.

\section{Confocal microscopy}

Tissue preparation

All confocal microscopy of rat tissues was performed under the approval of the University of Indiana IUCAC. The technique was modified from that developed previously for measurement of extracellular $\mathrm{pH}$ in colonic crypts by confocal imaging. ${ }^{18}$ Male Sprague- Dawley rats weighing 250 300 g (Charles River) were sacrificed with halothane vapour, and the oesophagus was excised, flushed, and muscle stripped. The isolated mucosal sheets were kept in ice cold Dulbecco's minimum Eagle's medium (Invitrogen, Carlsbad, California, USA). All tissue was used within two hours of isolation. The muscle stripped mucosa was mounted in a microscope chamber, permitting independent control of luminal and basolateral perfusates. The chamber was custom manufactured (Summit Precision Machining, Baltimore, Maryland, USA) and is a modification of our microscopy chamber for study of polarised functions in cultured epithelia. ${ }^{20}$ Perfusates were run continuously throughout experiments at $1 \mathrm{ml} / \mathrm{min}$ at room temperature, through luminal and basolateral chambers having volumes of $25 \mu \mathrm{l}$ and $15 \mu \mathrm{l}$, respectively. Perfusates for luminal perfusion were based on Krebs solution, titrated to $\mathrm{pH} 7.4$ or $\mathrm{pH}$ 1.5. Basolateral perfusates were based on physiological saline containing in mM: $130 \mathrm{NaCl}, 5 \mathrm{KCl}, 2 \mathrm{CaCl}_{2}, 1 \mathrm{MgSO}_{4}, 25$ mannose, 20 HEPES, and 0.1 mM carboxy SNARF-1, pH 7.4 or pH 6.5.

\section{Measurement of interstitial $\mathrm{pH}\left(\mathrm{pH}_{\text {int }}\right)$ in vitro}

The method was adapted from previously published methodology developed for measuring extracellular $\mathrm{pH}$ in which SNARF-1 was validated for emission ratiometric measurements of $\mathrm{pH}$ in extracellular tissue spaces in gastrointestinal epithelia using confocal microscopy. ${ }^{18}{ }^{21}$ We used a Zeiss LSM510 confocal microscope with a 40× C-Apo water immersion objective lens. The apical chamber was perfused with $\mathrm{pH}$ 1.5 or 7.4 Krebs solutions. The basolateral chamber was perfused with $\mathrm{pH} 7.4$ or $\mathrm{pH} 6.5$ solutions containing $0.1 \mathrm{mM}$ SNARF-1. SNARF-1 excitation was by the $488 \mathrm{~nm}$ line of an $\mathrm{Ar}$ laser, with emissions collected simultaneously at $580(25) \mathrm{nm}$ and $640(30) \mathrm{nm}$, using two detectors. The fluorescence intensity ratio of $640 \mathrm{~nm} / 580 \mathrm{~nm}$ was used to calculate $\mathrm{pH}$ values based on an experimentally established calibration curves. ${ }^{19}$ SNARF-1 was not included in perfusates with $\mathrm{pH}<6.5$ due to lack of solubility at low $\mathrm{pH}$. Interstitial $\mathrm{pH}$ was calculated from a calibration curve determined in the confocal microscope with 0.1 mM SNARF-1 in NaCl media of pH 6.0-8.0. A single point calibration was performed for each experiment to standardise $\mathrm{pH}$ calculation for daily instrument settings.

\section{In vivo microscopy}

Animal preparation

All animal studies were conducted under protocols approved by the West Los Angeles VAMC IUCAC. In vivo microscopy was performed, as modified from prior studies of the stomach and duodenum ${ }^{1622}$ and from prior studies of $\mathrm{pH}_{\text {int }}$ in mouse tumours. ${ }^{23}$ Male Sprague Dawley rats weighing approximately 300 g (Harlan Laboratories, San Diego, California, USA) were fasted overnight but had free access to water. After urethane $(1.25 \mathrm{~g} / \mathrm{kg})$ anaesthesia, the rat was placed supine on a plastic stage. Body temperature was maintained at $36-37^{\circ} \mathrm{C}$ by a heating pad and heat lamp. A tracheal cannula was inserted and saline was continuously infused through the left femoral vein at a rate of $1.08 \mathrm{ml} / \mathrm{h}$ using a Harvard infusion pump. Arterial blood pressure was monitored via a catheter placed in the left femoral artery. The abdomen was opened via a $3 \mathrm{~cm}$ midline incision and the anterior wall of the forestomach and

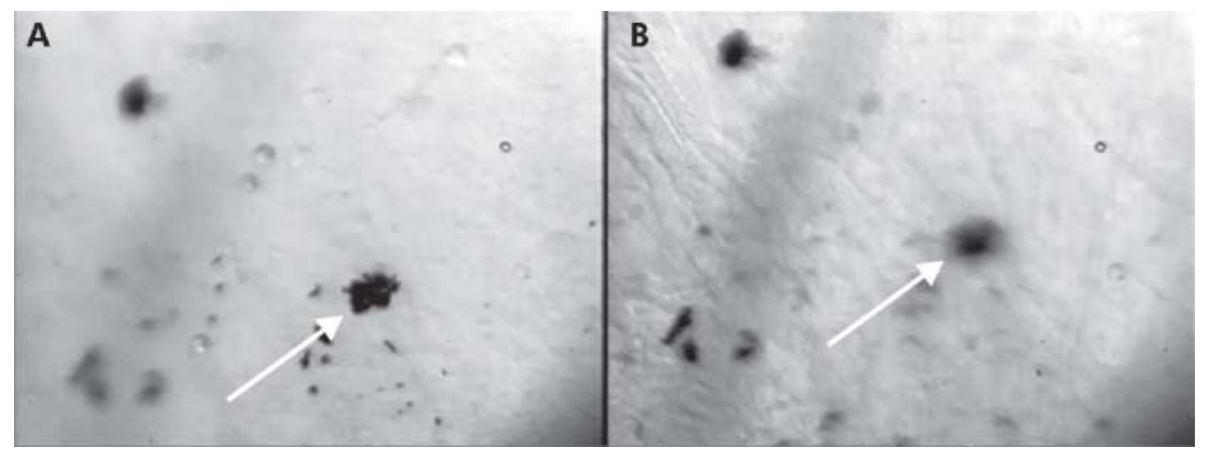

Figure 2 Optical measurement of oesophageal pre-epithelial layer thickness using carbon particles. A light rod was used to illuminate the mucosal surface at an acute angle in order to visualise the surface detail of the mucosa. Arrow indicates carbon particles on the luminal pre-epithelial layer surface. In $(\mathrm{A})$, the focal plane is at the pre-epithelial layer surface, at the level of the carbon particles. Note the cluster of carbon particles denoted by the white arrow. (B) The focal plane has been lowered to that of the mucosal surface. Note that the carbon particles cluster is out of focus whereas the mucosal surface is in focus. The $Z$ axis travel of the microscope between the two focal planes is considered to be equivalent to the thickness of the overlying pre-epithelial layer. 


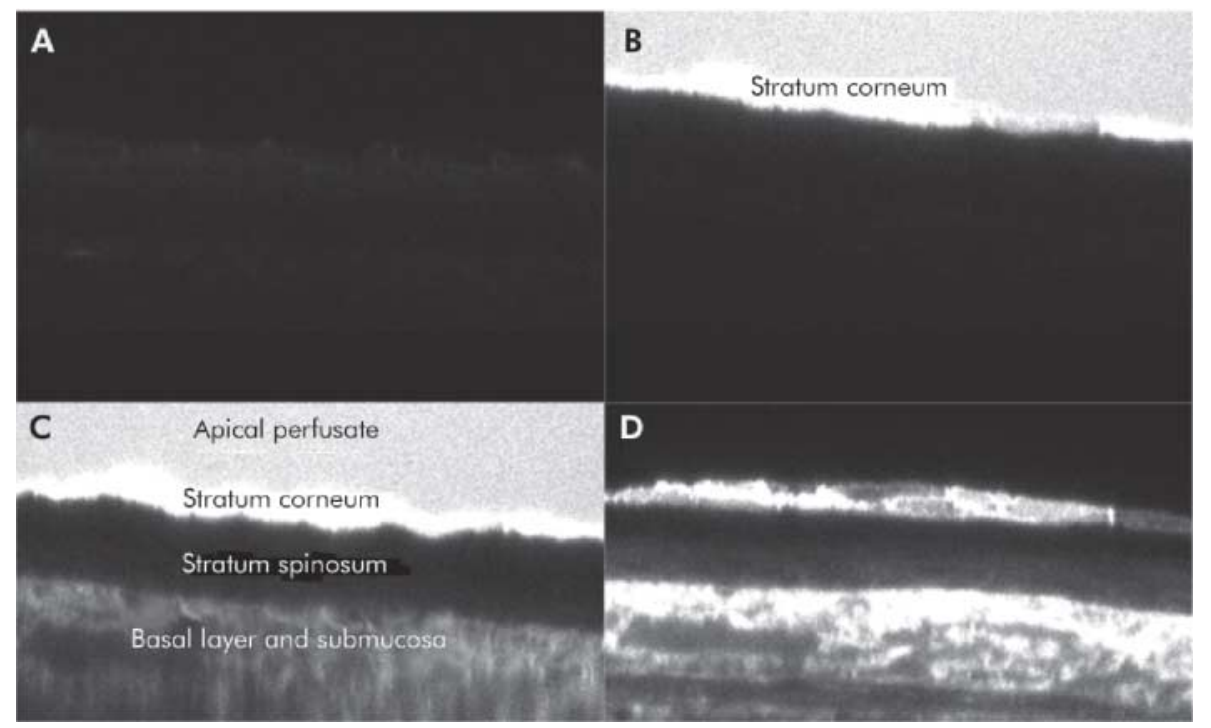

Figure 3 Oesophageal permeability to carboxy-seminapthorhodofluor-1 (SNARF-1). Confocal images of excised oesophagus in vitro depicted in the X-Z focal plane, for simultaneous imaging along the lumen to interstitium axis, were used to measure the depth of SNARF-1 permeation. All images were taken at $640 \mathrm{~nm}$ and $580 \mathrm{~nm}$ emission from the same tissue location at different times. (A) Image taken without SNARF-1. (b) Ten minutes' exposure to luminally perfused SNARF-1. Note the white staining of the superficial cells but absence of deeper penetration of the dye. (c) Ten minutes' exposure to luminally and serosally perfused SNARF-1. Note grey fluorescence of the lower pre-epithelial layers, and absence of penetration into the central pre-epithelial layer of the epithelium (black central band). (D) During washout with saline (no fluorescence in perfusate), SNARF-1 was retained in both fluorescent pre-epithelial layers.

lower oesophagus was incised using a miniature electrocautery. A concave stainless steel disk ( $16 \mathrm{~mm}$ diameter and 1.2 $\mathrm{mm}$ deep) with $3 \mathrm{~mm}$ central aperture was fixed watertight on the oesophageal mucosal surface with a silicone plastic adherent (Silly Putty; Binney and Smith Co., Easton, Pennsylvania, USA). A right angle laser Doppler probe was placed beneath the exposed oesophageal mucosa to measure oesophageal blood flow. A thin plastic coverslip was fixed to the disk with the silicone adherent to permit closed perfusion with solutions (total volume $50 \mathrm{ml}$; rate $0.25 \mathrm{ml} / \mathrm{min}$ ) using a Harvard infusion pump. After correctly placing the chamber on the mucosa, a small area of mucosa was isolated from extraneous secretion, and exposed only to perfusate, by virtue of being surrounded by silicone putty and being covered with the perfused chamber. Rats were injected with $0.5 \mathrm{ml}$ of $\mathrm{CF}$ $5 \mathrm{mg} / \mathrm{kg}$ intravenously in physiological saline five minutes before the experiments, as modified from published methodology. ${ }^{17}$

\section{Optical system}

For fluorescent microscopy, a modified multipurpose microscope (Carl Zeiss, Inc., Germany) with a 10× objective (NA 3.0; Rolyn Optics, Covina, California, USA) was used. The light source was a $100 \mathrm{~W}$ mercury lamp (Chiu Technical). A filter holder was placed in front of the lamp for manual replacement of 450 and $495 \mathrm{~nm}$ narrow bandpass excitation filters (Chroma Inc., Brattleboro, Vermont, USA). The television system consisted of a CCD colour camera (Optronics Engineering, Goleta, California, USA), a television monitor (Sony, Japan), and an S-VHS videotape recorder (Sony Electronics Inc., Japan). The CCD colour camera was connected to an Intel Pentium based IBM compatible microcomputer with FlashPoint framegrabbing videographic card (Integral Technologies, Inc., Silver Spring, Maryland, USA), and the captured image was digitised and stored on the hard disk of the computer for later analysis.

\section{Image analysis}

Fluorescent images of the microscopically observed chambered segment of oesophageal mucosa at $515 \mathrm{~nm}$ emission were captured and recorded using the computer hard disk drive. Capillary loops above the network of submucosal microvessels, which were assumed to be located in the subepithelial or basal epithelial layer of rat oesophagus, ${ }^{24}$ were used as the focal plane. Images were captured every five minutes. Emission intensity was measured 10 seconds before and after each designated time point. The paired readings needed to calculate a fluorescence ratio were thus taken at a maximum of 20 seconds apart. Image analysis was performed on the recorded images: three small areas of oesophageal submucosa between microvessels were selected at random and then followed throughout the experiment. Fluorescence intensity of the selected area was measured using an 8 bit image analyser software (Image-Pro Plus v. 1.3; Media Cybernetics, Silver Spring, Maryland, USA). The fluorescence intensity at $495 \mathrm{~nm}$ divided by that at $450 \mathrm{~nm}$ and the resulting ratio was converted to $\mathrm{pH}_{\text {int }}$ using an in vitro calibration curve according to the equation:

$$
\text { Fluorescence ratio }=\left(\mathrm{FI}_{495}-\mathrm{BGI}_{495}\right) /\left(\mathrm{FI}_{450}-\mathrm{BGI}_{450}\right)
$$

where FI=fluorescent intensity and BGI=background intensity.

BGI was defined as the intensity of oesophageal mucosa prior to CF loading. We found that this autofluorescence was homogeneous throughout the field and was close to 0 even with high camera gain and exposure time. BGI was therefore negligible compared with the high fluorescence of CF stained oesophageal mucosa. The mean of the ratios calculated from the three selected areas was defined as the ratio for that time period.

To demonstrate that in situ measurements were comparable with those measured in solution, in vitro tissue $\mathrm{pH}$ calibration was performed using conditions comparable with those in vivo experiments but with the use of muscle stripped oesophageal mucosa excised from sacrificed rats. Mucosae were equilibrated in CF free buffer solutions and were then placed in $5 \mu \mathrm{M}$ CF containing solutions at the same $\mathrm{pH}$ for 30 seconds. The tissue was then examined by fluorescence microscopy. Figure I depicts a typical calibration curve of the fluorescence ratio versus $\mathrm{pH}$ for the tissue $\mathrm{pH}$ calibration. The tissue calibration curve was similar to in vitro calibration 

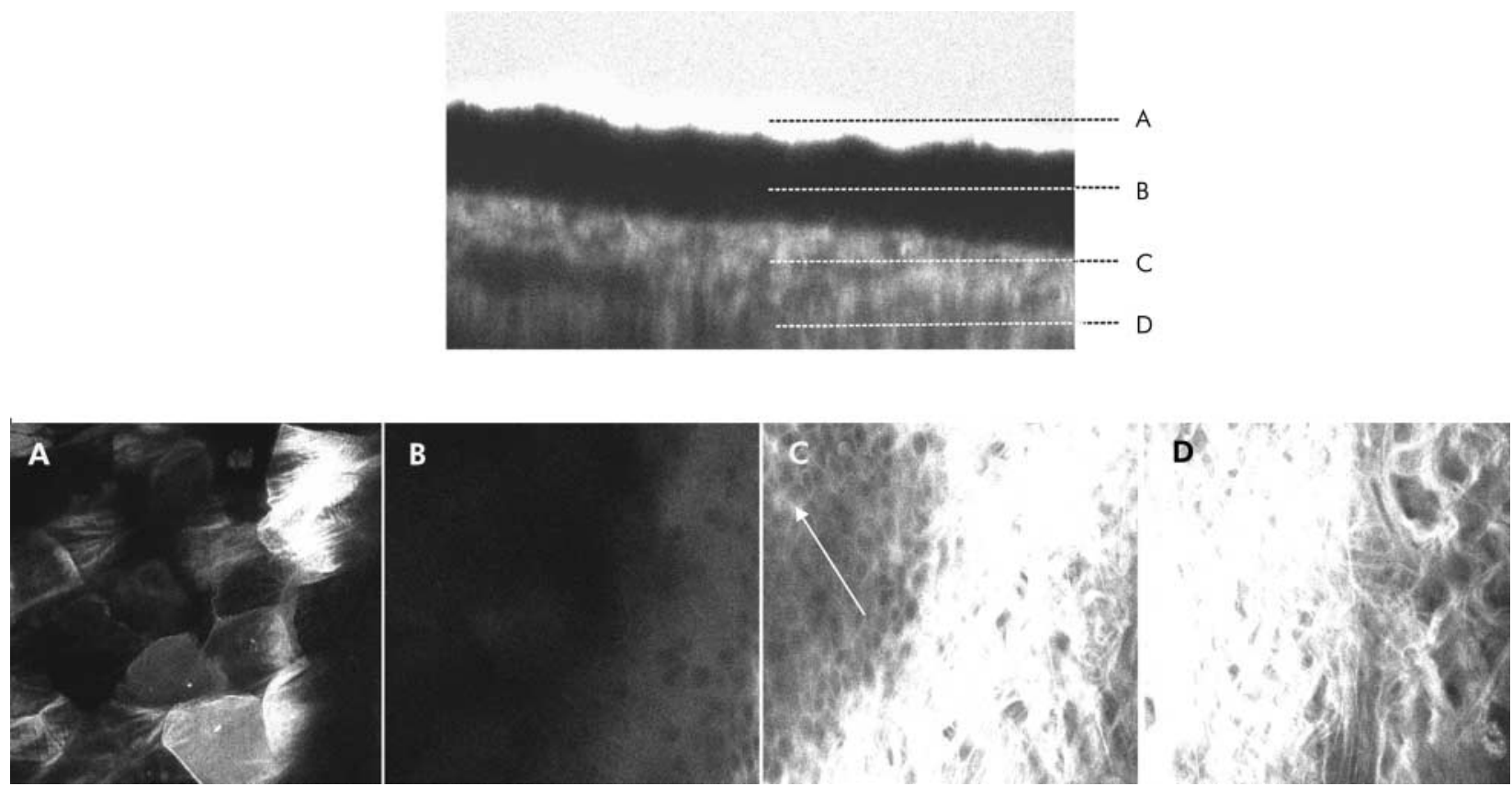

Figure $4 \quad X-Y$ plane confocal images of oesophageal mucosa following perfusion with carboxy-seminapthorhodofluor-1 (SNARF-1). Images were taken from a single area of the mucosa taken at different focal planes along the $\mathrm{Z}$ axis. A copy of fig $3 \mathrm{C}$ is included to orient the subsequent images. For image (A), the focal plane was at the epithelial surface (stratum corneum); for (B), the stratum spinosum; for (C), the basal cell pre-epithelial layer; and in (D), the subepithelial connective tissue. Note that SNARF-1 fluorescence, which appears white, was taken up by the flattened polygonal surface cells, indicating that these cells are non-viable, and also permeated the interstitial space in the basal cell and subepithelial pre-epithelial layers, as seen by a honeycomb-like pattern in the left potion of (C) (arrow), but did not permeate the stratum spinosum (B).

curves constructed using aqueous solutions containing $5 \mu \mathrm{M}$ $\mathrm{CF}$ in Krebs solutions at varying $\mathrm{pH}$, as described previously. ${ }^{16} 17$

\section{Measurement of pre-epithelial layer thickness}

Pre-epithelial layer thickness was measured using a modification of previously described techniques. ${ }^{25}{ }^{26}$ Graphite particles were placed over the mucosa to delineate the luminal surface of the pre-epithelial layer. The oesophageal mucosal surface was visualised with a fibreoptic light rod (American Optical Scientific Instruments, Buffalo, New York, USA) at $45^{\circ}$ from the mucosa in order to provide reflectance images of the epithelial surface (fig 2 ). The microscope was alternately focused from the oesophageal surface to the graphite pre- epithelial layer. The distance of vertical travel of the microscope objective was measured by using a digital $\mathrm{Z}$ axis measuring device (Quick-Check, Metronics, Bedford, New Hampshire, USA) connected to the microscope, providing a measure of pre-epithelial layer thickness.

\section{Measurement of oesophageal blood flow}

We simultaneously measured oesophageal blood flow and $\mathrm{pH}_{\mathrm{in}}$ using a technique modified from our prior measurements of blood flow and $\mathrm{pH}_{\mathrm{i}}$ in gastric and duodenal epithelia. ${ }^{16}{ }^{27} \mathrm{~A}$ right angle probe (\#H41-3667; Vasomedics Inc., St. Paul, Minnesota, USA) was placed beneath the oesophageal mucosa. Blood flow was measured as the voltage output of the laser Doppler instrument (LaserFlo BPM803A; Vasomedics Inc.) and was expressed relative to basal measurements made during the last 4-5 minutes of the dye loading period.

\section{Acid perfusion protocol}

Oesophageal mucosae were superfused with $\mathrm{pH}$ 7.0 Krebs for 15 minutes, after which the perfusate was changed to $\mathrm{pH} 1.0$ for 40 minutes. In some rats, indomethacin pretreatment $(5$ $\mathrm{mg} / \mathrm{kg}$ subcutaneously) was given 30 minutes before acid perfusions.

\section{Statistics}

All data are expressed as mean (SEM). Comparisons between groups were made by one way analysis of variance (ANOVA) followed by Fisher's least significant difference test. A p value of less than 0.05 was taken as significant.

\section{RESULTS}

\section{In vitro measurements of oesophageal $\mathrm{pH}_{\mathrm{int}}$}

We used confocal microscopy of dye loaded oesophagus in vitro to localise the fluorescent dye reporting $\mathrm{pH}_{\mathrm{int}}$. SNARF-1 was chosen due to its $\mathrm{pK}_{\mathrm{a}}$ and physical properties that resemble fluorescein dyes, and because of its dual emission characteristic that can be used with confocal microscopy. ${ }^{19}{ }^{28}$ An in vitro preparation was used to facilitate perfusion of the basolateral surface. Figure 3 depicts fluorescence distribution in the confocal X-Z plane (optical cross section) using images of rat oesophageal mucosa from the same tissue location under several conditions: before dye perfusion (fig 3A) and subsequently after either luminal (fig 3B) or luminal plus serosal (fig 3C) addition of SNARF-l dye and 10 minutes following removal of dye from both perfusates (fig 3D). Dye uptake occurred in an intensely fluorescent (white appearing) band when exposed to luminal SNARF, and had a more mottled appearance when SNARF was perfused over the basolateral surface of the preparation. Notably, SNARF was excluded from the portion of the epithelium farthest from the luminal or serosal surfaces (black band between fluorescent areas). As seen, dye introduced from the serosal perfusate permeated the intercellular space between the basal epithelial cells. To further localise the dye, serial X-Y (plane of the oesophageal mucosa) confocal sections were obtained in tissue 10 minutes after perfusion with SNARF-1 over the luminal and serosal mucosal surfaces (fig 4). A copy of fig 3C is included for orientation, with broken lines depicting the focal planes for each subsequent panel of fig 4 . Note that SNARF-1 stained the flat polygonal cells of the stratum corneum (fig 4A), did not stain the middle epithelial layer (fig 

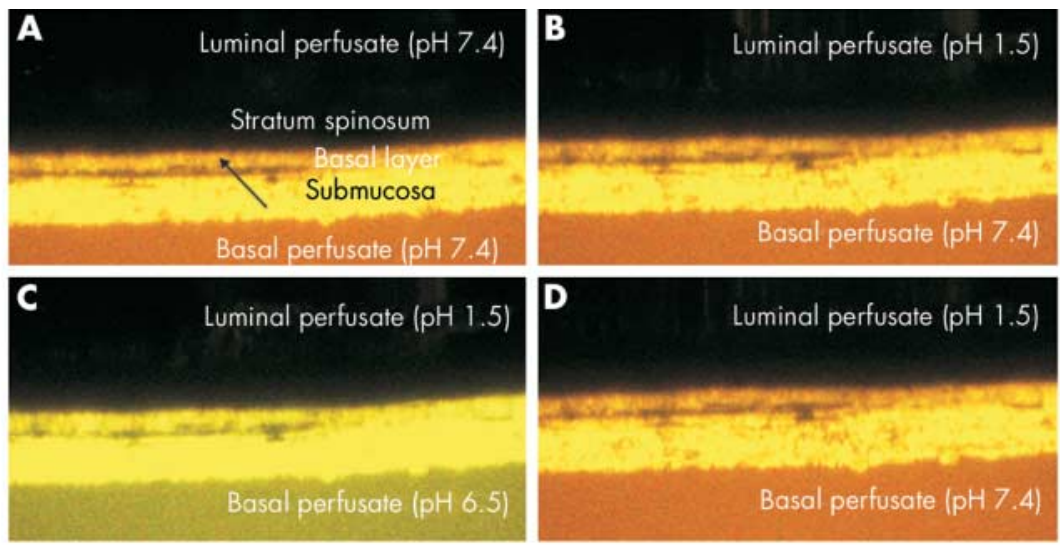

A

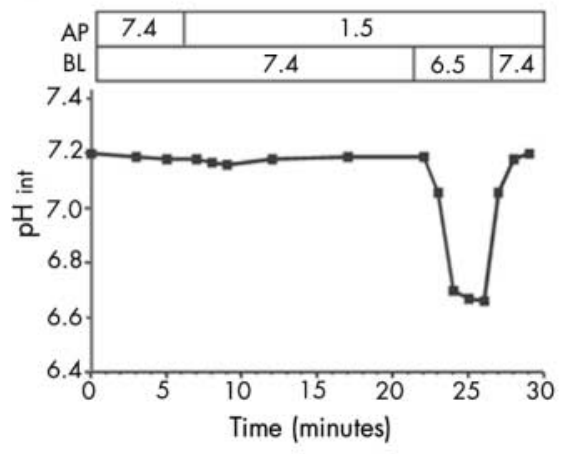

B

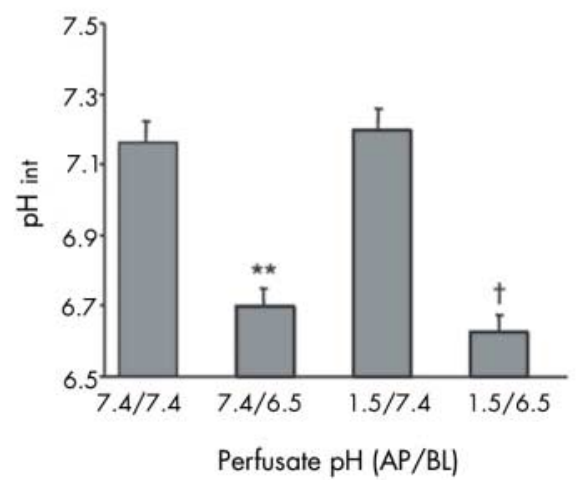

Figure 5 Interstitial $\mathrm{pH}\left(\mathrm{pH}_{\text {int }}\right)$ of the basal pre-epithelial layer of rat oesophagus measured with carboxy-seminapthorhodofluor-1 (SNARF-1). $\mathrm{X}$-Z images were taken from a single tissue location. Colours in the images correspond to $\mathrm{pH}$ (green-acid; red-alkaline). The arrow indicates interstitial fluorescence in the basal pre-epithelial layer from which $\mathrm{pH}_{\text {int }}$ was measured. Top: (A) With basolateral and apical perfusates $\mathrm{pH}=7.4$, the $\mathrm{pH}_{\text {int }}$ of the basal pre- epithelial layer is alkaline (yellow); $(\mathrm{B})$ acidifying the luminal perfusate to $\mathrm{pH} 1.5 \mathrm{did}$ not affect $\mathrm{pH}$ int of the basal pre-epithelial layer; $(\mathrm{C})$ acidifying the basal pre-epithelial layer to $\mathrm{pH} 6.5$ acidified the basal pre-epithelial layer, as manifest by the green fluorescence; (D) $\mathrm{pH}_{\text {int }}$ of the basal pre-epithelial layer recovered after the $\mathrm{pH}$ of the basolateral perfusate was changed to $\mathrm{pH} 7.4$, despite the apical perfusate $\mathrm{pH}$ of 1.5. Bottom: (A) time course of $\mathrm{pH}_{\text {int }}$ for one of the experiments. $\mathrm{AP}, \mathrm{pH}$ of apical; $\mathrm{BL}$, $\mathrm{pH}$ of basolateral perfusions. (B) Data summary $(n=6)$. ${ }^{*} \mathrm{p}<0.01$ versus perfusion with $\mathrm{AP} / \mathrm{BL} \mathrm{pH} 7.4 / 7.4 ;+\mathrm{p}<0.01$ versus perfusion with $\mathrm{pH} 1.5 / 7.4$.

$4 \mathrm{~B})$, but when applied to the basolateral surface stained the basal cell layer and submucosa (fig 4C, 4D, respectively). These data are consistent with SNARF-1 penetrating the outermost 2-3 pre-epithelial layers of the stratum corneum and entering the flat polygonal cells of the outermost layer. The high SNARF-1 permeability of these outer cells suggests they have compromised viability, or at least compromised membrane integrity. In contrast, SNARF-1 staining at the basal layer appears to be exclusively extracellular, with dye permeating only the lateral spaces between basal cells (fig 4C, arrow). Staining of submucosal tissue in fig $4 \mathrm{D}$ suggests that SNARF may be adhering to certain proteins in the extracellular matrix, potentially explaining the retention of fluorescence after removal of SNARF-l from the perfusates. Notably, dye was completely excluded from the remainder of the epithelium which, by inference, is the stratum spinosum. After localising the dye to the basal pre-epithelial layer, we then imaged this pre-epithelial layer in the presence of acid perfused over the apical and basolateral mucosal surfaces.

Figure 5 depicts SNARF-1 emission ratio images in the X-Z focal plane after perfusion of the basolateral surface with SNARF-1 for 10 minutes. Pseudocolour (green-acid; redalkaline) corresponds to $\mathrm{pH}_{\mathrm{int}}$. SNARF-1 could not be added to the apical perfusate as it precipitates at strongly acidic $\mathrm{pH}$. Figure 5A, 5B, and 5D qualitatively demonstrate lack of change in $\mathrm{pH}_{\mathrm{int}}$ when luminal $\mathrm{pH}$ is changed from $\mathrm{pH} 7.4$ to 1.5. This is quantitatively confirmed by the time course experiment shown in the bottom curves (A) and (B). Ability to elicit and detect changes in $\mathrm{pH}_{\text {int }}$ in the basal layer is demonstrated by perfusion with a mildly acidic ( $\mathrm{pH} 6.5)$ solution in the basolateral chamber (fig 5C and bottom cures (A) and (B)). These data are consistent with the lack of transmucosal acid permeation even in the presence of physiological transmucosal acid gradients.

\section{Measurements of oesophageal $\mathrm{pH}_{\text {int }}$ in vivo}

In order to measure the depth of acid penetration into the mucosa in our in vivo model, we measured $\mathrm{pH}_{\text {int }}$ using a technique in which CF (free acid), injected intravenously, leaked into the interstitial space. CF was used in preference to the structurally related SNARF-1 because our fluorescence microscope used for in vivo studies was equipped only for excitation ratio measurements, necessitating the use of a fluorophore appropriate for these measurements, such as CF. We continuously observed the mucosa under transillumination, injected CF intravenously, and then observed the development of fluorescence. As seen in fig 6, the submucosal vessels fluoresced shortly after CF injection, with transfer of fluorescence out of the vascular space occurring within 30 seconds after CF injection. Overall, CF fluorescence reached a maximum at 20 minutes, then gradually decreased, and remained at a level sufficient to obtain reproducible fluorescence ratios for 60 minutes, with a stable fluorescence intensity ratio $\left(\mathrm{F}_{495} / \mathrm{F}_{450}\right)$ during perfusion with $\mathrm{pH} 7.0$ buffer (fig 7). The ratio however decreased 

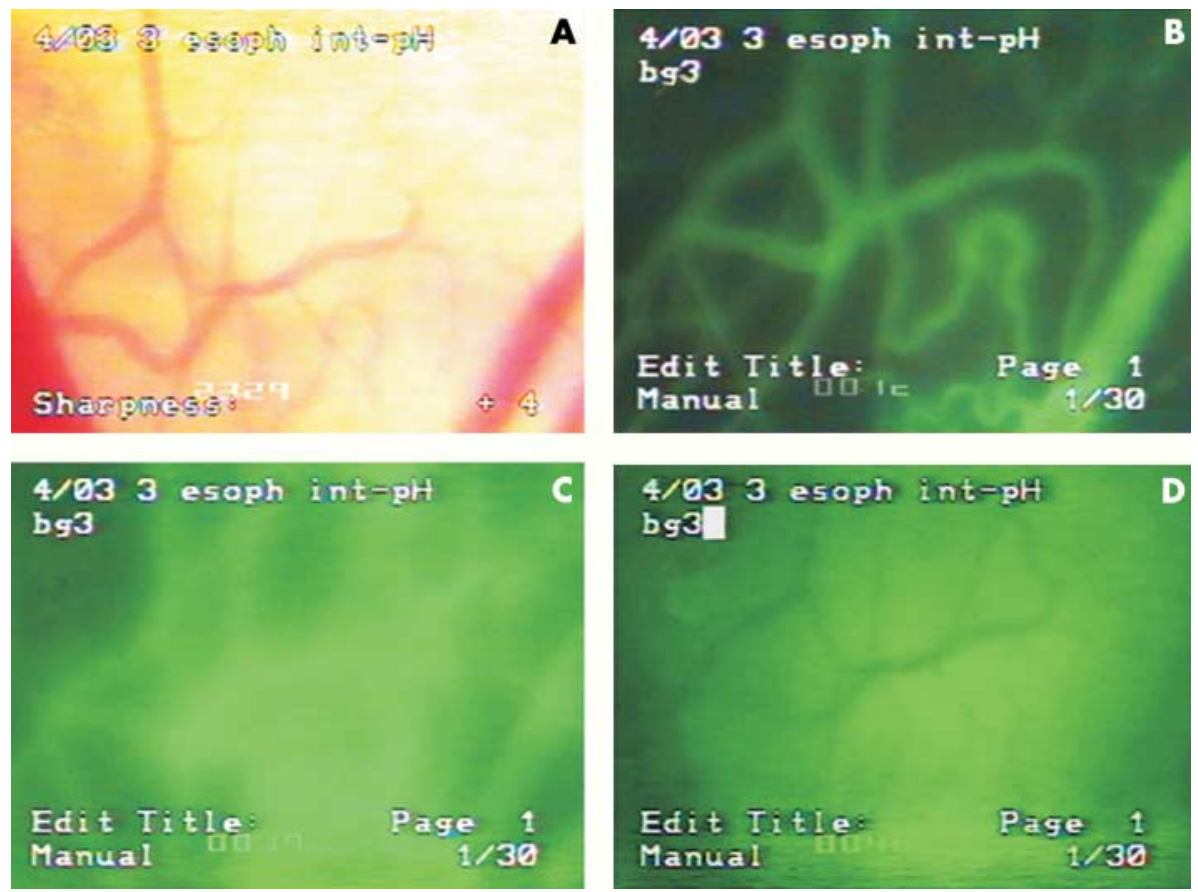

Figure 6 Microscopic image of oesophageal mucosa demonstrating interstitial extravasation of intravenous 5-(and-6)-carboxyfluorescein (CF). (A) Transillumination demonstrates submucosal blood vessels. (B) Twelve seconds after CF injection intravenously the submucosal vessels fluoresce under $495 \mathrm{~nm}$ excitation. The fluorescence appears to be confined to the intravascular space. (C) Nineteen seconds after injection, extravasation is seen in the vicinity of the vessels. (D) Forty eight seconds after injection, the interstitium fluoresces whereas the vessels appear dark, indicating complete extravasation of the circulating dye into the interstitium.

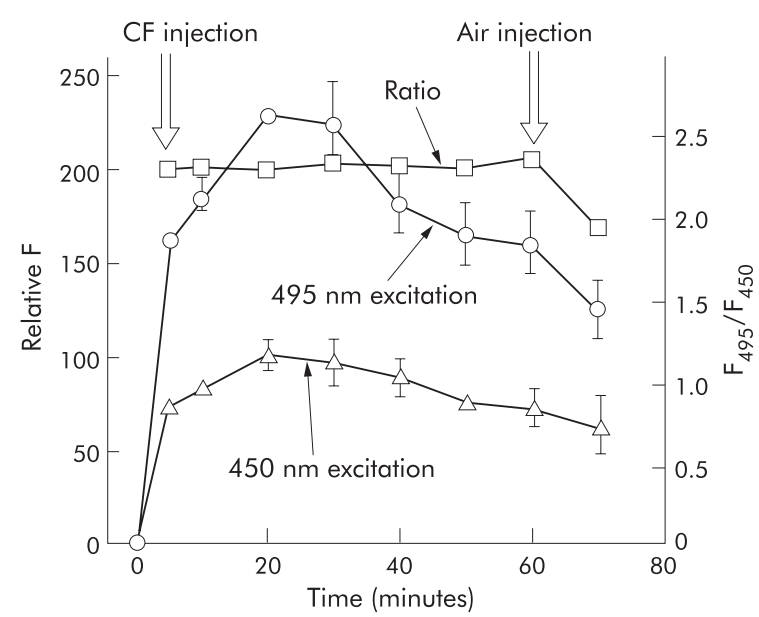

Figure 7 Time course of fluorescence intensity of oesophageal mucosa measured at $530 \mathrm{~nm}$, with excitation alternately at 450 and $495 \mathrm{~nm}$. Note that fluorescence increased and then decreased over time when the tissue was excited at either wavelength. The ratio calculated from the fluorescence measurements was stable, consistent with a stable $\mathrm{pH}_{\mathrm{int}}$. Air injection at 60 minutes lowered the ratio, consistent with the development of interstitial acidosis. CF, 5(and-6)-carboxyfluorescein.

10 minutes after the rats were killed by intravenous air injection at 60 minutes. Basal $\mathrm{pH}_{\text {in, }}$ measured during perfusion with $\mathrm{pH} 7.0$ buffer perfusion, was $7.25(0.05)(\mathrm{n}=22)$. As shown in fig 8, rat oesophageal $\mathrm{pH}_{\mathrm{int}}$ was unchanged during perfusion of luminally applied $\mathrm{pH} \quad 1.0$ solution, further supporting our hypothesis that the oesophageal epithelium is an effective barrier to acid diffusion.

As prostaglandins enhance mucosal injury in experimental oesophagitis models ${ }^{29}{ }^{30}$ in contrast with the gastroduodenal mucosa, ${ }^{31}$ we examined the effect of indomethacin pretreatment on $\mathrm{pH}_{\text {int }}$ during perfusion with $\mathrm{pH} 7.0$ or 1.0 solutions on

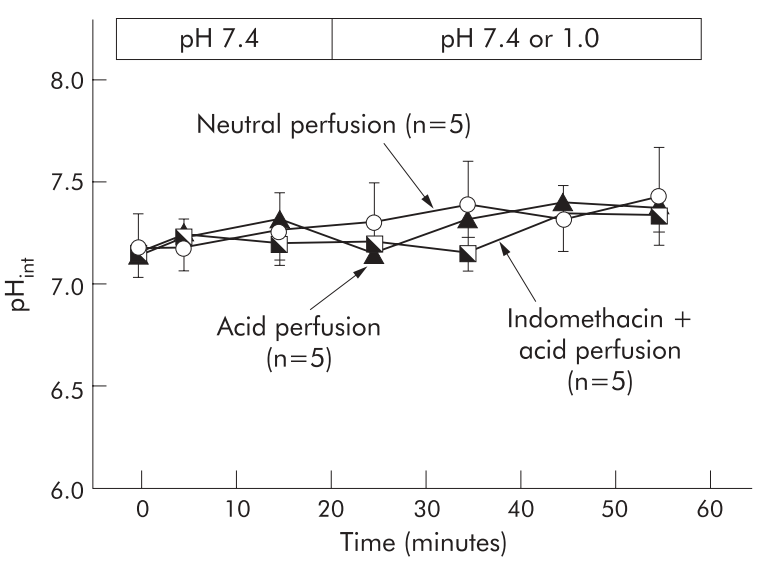

Figure 8 Effect of perfusion of the luminal surface with acid and with indomethacin intraperitoneally on $\mathrm{pH}_{\text {int }}$ of oesophageal mucosa measured in vivo. Note that neither intervention affected $\mathrm{pH}_{\text {. }}$

consistent with failure of acid to permeate from the luminal surface to the vassal pre-epithelial layer, where the dye was present to report $\mathrm{pH}_{\text {int. }}$

oesophageal defence mechanisms in the absence of injury. Indomethacin had no effect on $\mathrm{pH}_{\mathrm{int}}$ in our system.

\section{Measurement of pre-epithelial layer thickness}

We then measured changes in relative pre-epithelial layer thickness to determine if it increased during luminal acid perfusion, suggestive but not conclusively implicating a protective role for the surface pre-epithelial layer. Pre-epithelial layer thickness varied from 0 to $67 \mu \mathrm{m}$, with a mean thickness of 29 (2) $\mu \mathrm{m}(\mathrm{n}=67,2-3$ measurements/rat $)$, and did not increase immediately in response to acid perfusion. Due to the delayed and gradual increase in pre-epithelial thickness, we were only able to demonstrate a statistically significant increase in pre-epithelial thickness after a 40 minute perfusion with $\mathrm{pH}$ 


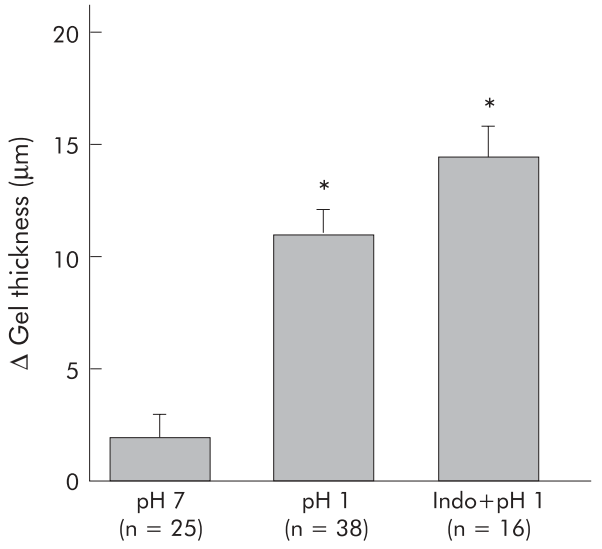

Figure 9 Change in the thickness of the pre-epithelial layer overlying the oesophageal mucosa. The pre-epithelial layer was significantly thickened during $\mathrm{pH} 1$ perfusion but was not affected by indomethacin (Indo) intraperitoneally. ${ }^{*} \mathrm{p}<0.05$ versus $\mathrm{pH} 7$ perfusion by ANOVA.

1.0 solution $(\mathrm{p}<0.05)$ compared with the thickness increase during perfusion with a pH 7.0 solution (fig 9).

\section{Measurement of oesophageal blood flow}

Hyperaemia during luminal acid challenge is another means by which gastrointestinal mucosa are protected during acid perfusion. ${ }^{32}{ }^{33}$ We thus measured blood flow in the oesophagus using laser Doppler flowmetry. Figure 10 depicts relative oesophageal mucosal blood flow in rats perfused with either neutral or acidic solutions. In control rats perfused with neutral solutions, relative mucosal blood flow gradually decreased to $88(5) \%$ of baseline $(n=7)$. During acid perfusion, mucosal blood flow significantly increased compared with control rats $(\mathrm{p}<0.05)$. Pretreatment with indomethacin had no effect on this increase in blood flow. This increased blood flow indicates that submucosal structures such as blood vessels can be influenced by luminal acid, in the absence of transepithelial acid diffusion, consistent with an indirect transduction mechanism. Increased blood flow and pre- epithelial layer thickness in response to acid is reminiscent of the gastroduodenal protective response to luminal acid.

\section{DISCUSSION}

We have demonstrated that intravenously injected water soluble $\mathrm{pH}$ indicating fluorescent dyes can permeate the interstitial space of the basal pre-epithelial layer of the rat oesophagus, serving as useful markers for acid penetration into the mucosa. We found that the $\mathrm{pH}_{\mathrm{int}}$ of the basal layer of the rat oesophagus was unchanged when the luminal surface was exposed to high luminal acidity in vitro and in vivo, although $\mathrm{pH}_{\mathrm{int}}$ decreased during exposure to modestly acidic solutions exposed to the basolateral surface of the preparation. We also found that pre-epithelial layer thickness and blood flow increased in response to luminal acid in rat oesophagus in vivo. We could not find any effects of indomethacin on our measurements.

The use of fluorophores to measure $\mathrm{pH}_{\mathrm{int}}$ has been described in the literature for several decades. In our case, we used two dyes, both of which are highly water soluble and thus excluded from viable cells having an intact plasma membrane. These dyes, as shown by others, readily migrate from the vascular space or from a mucosal bathing solution to the intercellular interstitium, where they can faithfully record $\mathrm{pH}_{\text {int }}$. The emission light path of confocal microscopes is split, enabling simultaneous imaging of the same field at two wavelengths. Dyes such as SNARF-1 take advantage of this feature as they have a $\mathrm{pH}$ dependent measurable shift of emission wavelength. Conventional epifluorescent microscopes, such as used

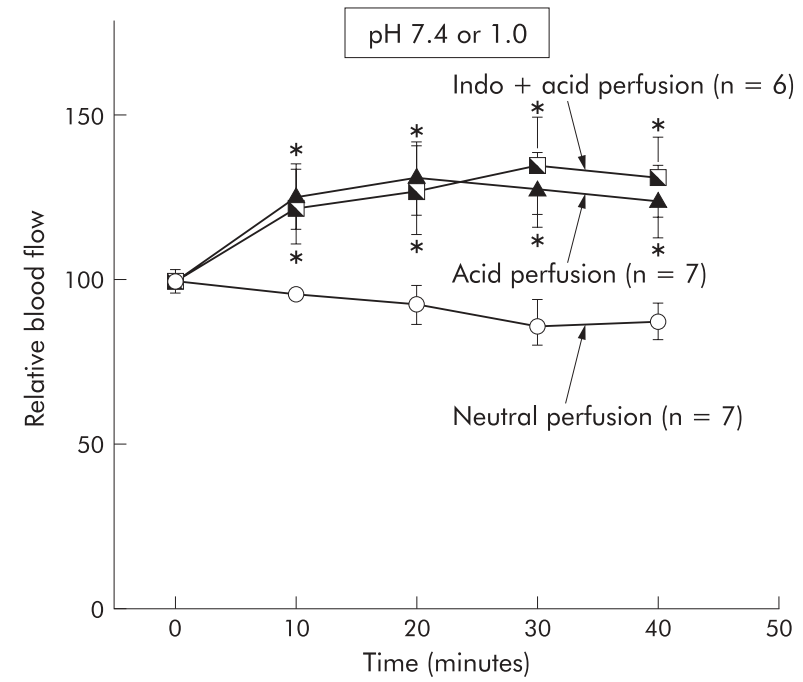

Figure 10 Relative blood flow of oesophageal mucosa. Acid perfusion increased significantly mucosal blood flow but indomethacin (Indo) did not affect this acid induced increase. ${ }^{*} \mathrm{p}<0.05$ versus $\mathrm{pH} 7$ perfusion by ANOVA.

for the in vivo studies, do not have emission beam splitters. Hence fluorophores such as CF are used, in which the $\mathrm{pH}$ dependent ratio of fluorescence intensity at a single wavelength is measured while alternating between two excitation wavelengths. This is the more usual method used for FRIM based pH measurements (fluorescence ratio intensity measurement).

Measurements of $\mathrm{pH}_{\text {int }}$, pre-epithelial layer thickness, and blood flow all reflect the response of the epithelium to acid challenge. The 40-70 minute measurement period used for the in vivo studies is comparable with the duration of acute clinical reflux episodes ${ }^{34}$ although clinical disease is thought to develop after months or years of repeated acute reflux episodes. Through these types of measurements, we have been able to study mucosal physiology to understand which factors need to be regulated in order to prevent injury. In this fashion, these studies complement those designed with injury as an end point. We measured the $\mathrm{pH}_{\mathrm{int}}$ of rat oesophagus with two novel but related methods: an in vivo microscopic technique and an in vitro confocal microscopic technique. Unexpectedly, changes in $\mathrm{pH}_{\mathrm{int}}$ in the basal epithelial layer were not demonstrated, in spite of high luminal acidity. Basal interstitial pH decreased only when the blood supply was stopped by intravenous air injection in vivo or when the basolateral perfusate was changed from neutral to $\mathrm{pH} 6.5$ solution in vitro. These findings indicated that the $\mathrm{pH}_{\mathrm{int}}$ of the basal epithelial region was largely dependent on the $\mathrm{pH}$ of the blood or basolateral fluid rather than the $\mathrm{pH}$ of the lumen, and were consistent with low acid permeability of the outer epithelial layers to acid.

Oesophageal epithelial resistance to luminal acid has been extensively studied by Orlando et al, in addition to other investigators. The rabbit oesophageal epithelium is electrically tight (1000-2500 $\Omega \times \mathrm{cm}^{2}$ ), with contributory structural components located predominantly in the stratum corneum and upper stratum spinosum. ${ }^{9}$ Prolonged contact with luminal acid and pepsin alters the properties of the intercellular junctions so as to increase paracellular pathway permeability, ${ }^{15}{ }^{35}$ enabling influx of protons into the intercellular space and subsequent mucosal acidification. Acidification of the intercellular space (that is, low $\mathrm{pH}_{\mathrm{int}}$ ) is important in that the oesophageal epithelial cell serosal membrane has a much greater susceptibility to acid injury than the apical membrane. ${ }^{1536}$ Protons must have penetrated the mucosa to some degree as luminal 
acid perfusion increased oesophageal blood flow. Furthermore, staining of the cytoplasm of the cells of the stratum corneum (fig 4) indicates that there was acid penetration into the most superficial portion of the epithelium, where dye penetration into the cytoplasm indicated the presence of non-viable cells. Nevertheless, the degree of penetration, as judged from the in vivo and in vitro studies, was superficial as we were unable to acidify the basal stratum by exposing the luminal surface to $\mathrm{pH}$ 1.0, although it is possible that acid penetrated into the stratum spinosum where no dye was present to report $\mathrm{pH}_{\mathrm{inn}}$. That no dye was present in the stratum spinosum also underscored the apparent impenetrability of this epithelial layer. These observations are thus consistent with intercellular dilution and buffering of back diffusing $\mathrm{H}^{+37}$ or, more likely, the impermeability of the stratum spinosum to aqueous solutes, including protons. Coupled with the increase in blood flow (and possibly of pre-epithelial layer thickness) in response to luminal acid perfusion, these data are consistent with mucosal responses to luminal acid being mediated by neural acid sensing mechanisms, as has been observed previously with the stomach and duodenum, ${ }^{27}{ }^{38}$ or by non-neural pathways, rather than from direct penetration of acid through the epithelium into the basal pre-epithelial layer. These sensing mechanisms have been suspected on the basis of the ability of subjects with reflux disease to sense the presence of acid perfused into the oesophageal lumen, ${ }^{39}$ in addition to the aforementioned mucosal responses to luminal acid perfusion. Nevertheless, available immunohistochemical data have not been consistent with penetration of afferent nerves into epithelial strata more superficial than the basal cell layer. ${ }^{40}$ This raises the question as to how a luminal acid signal is transduced in the absence of proton permeation through the mucosa to the location of the afferent nerves. Although precedents exist for local signal transmission via extracellular local mediators (for example, $\mathrm{Ca}^{++}$, adenosine) and intracellularly via gap junctions, in the absence of data we can only speculatively present these possibilities.

Only a few studies have addressed the presence of and thickness of the oesophageal pre- epithelial layer. The thickness of the unstirred pre-epithelial layer overlying the oesophagus, thought by some to be comparable with pre-epithelial layer thickness ${ }^{41}$ has been estimated to be 30 $\mu \mathrm{m},{ }^{42}$ close to our results, although another group reported that an adherent pre- epithelial layer was absent in normal rat oesophagus. ${ }^{43}$ Unlike other species, rats do not have oesophageal submucosal glands. Therefore, the pre-epithelial layer in the rat oesophagus may be derived from either salivary glands or gastric juice by reflux, as has been suggested by several groups, ${ }^{54}$ although our closed system would prevent these factors from further influence on the pre-epithelial layer. Another possibility is that the pre-epithelial layer is derived from the keratinocyte cornified envelope, the protein-lipid polymer that is thought to form the watertight outer barrier of all squamous epithelia, ${ }^{45}$ although the optical properties of the cornified layer are not known. Surprisingly, the thickness of the pre-epithelial layer increased in response to luminal acid, as we have previously described for the mucous layer in rat duodenum. ${ }^{26}$ As there are few data supporting the presence of alkaline secretion or mucin secretion in the oesophagus of species devoid of submucosal glands, such as the rat and rabbit, compared with the gland bearing oesophagus of opossums and humans ${ }^{46}$ it is likely that the observed pre-epithelial layer is not composed of mucus, as is the case in the duodenum. Nevertheless, there is evidence that the oesophagus-like stratified squamous epithelial cells of the larynx, vagina, and the ocular surface produce mucin-like glycoproteins or glycoconjugates ${ }^{97}$ in the absence of specialised goblet cells. ${ }^{48}$ The mechanisms responsible for the increased thickness in response to luminal acid in this study however remain unknown.

Luminal acid significantly increased oesophageal blood flow, similar to the oesophageal blood flow responses to lumi- nal acid measured in rabbits and cats. ${ }^{49}$ As the rat oesophagus does not secrete $\mathrm{HCO}_{3}^{-}$, the significance of this hyperaemia is uncertain since there are few data supporting the presence of acid back diffusion through the normal oesophagus, ${ }^{50}$ unlike the readily demonstrable acid back diffusion in the stomach and duodenum..$^{50}{ }^{51}$ Duda et al demonstrated that prostaglandins increased oesophageal blood flow in rabbits. ${ }^{52}$ However, pretreatment with indomethacin did not abolish the acid induced increase in $\mathrm{BF}$ in this study, indicating that prostaglandins were not involved in the observed blood flow response. Recent investigations revealed that capsaicin sensitive afferent nerves and calcitonin gene related peptide mediated increased oesophageal blood flow in response to luminal noxious agents such as ethanol and bile acid..$^{53} \mathrm{As}_{\mathrm{pH}}$ int $\operatorname{did}$ not decrease during acid induced hyperaemia, back diffused acid may be buffered before reaching the basal epithelial layer, or may more likely result from release of vasodilatory peptides and nitric oxide in response to neural activation of epithelial acid receptors, as has been shown in the stomach and duodenum, ${ }^{27}$ and recently in the oesophagus. ${ }^{54}$ Lastly, the hyperaemic response to acid perfusion may be a consequence of the increased contractile activity of the oesophageal smooth muscle, as suggested by Hollwarth and colleagues. ${ }^{49}$ Although pre-epithelial layer thickness and blood flow both respond to luminal acid perfusion, their role in the overall defence of the oesophageal mucosa from acid related injury remains conjectural.

Endogenously generated prostaglandins have been convincingly demonstrated to protect the stomach and duodenum from acid induced injury. ${ }^{556}$ This paradigm however may not apply to the oesophagus in particular as indomethacin, a non-selective cyclooxygenase inhibitor, can have salutary effects on oesophageal protective mechanisms, ${ }^{29} 3057$ in contrast with its effects on the stomach and duodenum. Our data provide more support for this paradigm as indomethacin did not adversely affect measured defence mechanisms.

In summary, the rat oesophageal epithelium is highly resistant to acidification from prolonged exposure to concentrated mineral acid presented to the apical surface. Despite this lack of acidification, unknown mechanisms transduce the presence of luminal acid into physiological responses such as increased blood flow and increased pre-epithelial layer thickness. It is likely that an impermeable structure combined with acid sensing neural mechanisms and a separate transepithelial transduction mechanism underlie the observed mucosal acid responses that occur despite the remarkable acid resistance of the rat oesophagus. Further understanding of these early acid responses could lead to new therapeutic targets for therapies designed to prevent the acid induced mucosal damage that underlies advanced oesophageal patho$\log$.

\section{ACKNOWLEDGEMENTS}

We would like to thank Jonathan Lee for his technical assistance. Supported by Department of Veterans Affairs Merit Review funding, and NIH-NIDDK ROI DK54221, 5P30DK041301, and RO1 DK54940.

\section{Authors' affiliations}

S Tanaka*, M Hirokawa, J D Kaunitz, West Los Angeles Veterans Affairs Medical Centre, CURE: Digestive Diseases Research Centre, and Department of Medicine, School of Medicine, University of California Los Angeles, Los Angeles, CA, 90073, USA

S Chu, M H Montrose, Department of Physiology, Indiana University, School of Medicine, Indianapolis, Indiana, 46202, USA

*Present address: Department of Gastroenterology, National Tokyo Medical Centre, 2-5-1 Higashigaoka, Meguro-ku, Tokyo 152-8902, Japan

\section{REFERENCES}

1 Fitzgerald RC, Omary MB, Triadafilopoulos G. Dynamic Effects of acid on Barrett's esophagus. An ex vivo proliferation and differentiation model. J Clin Invest 1996;98:2120-8. 
2 Fitzgerald RC, Omary MB, Triadafilopoulos G. Altered sodium-hydrogen exchange activity is a mechanism for acid-induced hyperproliferation in Barrett's esophagus Am J Physiol Gastrointest Liver Physiol 1998;275:G47-55.

3 Bateson MC, Hopwood D, Milne G, et al. Oesophageal epithelia ultrastructure after incubation with gastrointestinal fluids and their components. J Pathol 1981;133:33-51.

4 Salo JA, Lehto VP, Kivilaakso E. Morphological alterations in experimental esophagitis. Light microscopic and scanning and transmission electron microscopic study. Dig Dis Sci 1983;28:440-8.

5 Sarosiek J, McCallum RW. Mechanisms of oesophageal mucosal defence. Baillieres Best Pract Res Clin Gastroenterol 14:701-17.

6 Abdulnour-Nakhoul S, Nakhoul NL, Orlando RC. Lumen-to-surface pH gradients in opossum and rabbit esophagi: role of submucosal glands. Am J Physiol Gastrointest Liver Physiol 2000;278:G1 13-20.

7 Goldstein JL, Watkins JL, Greager JA, et al. The esophageal mucosal resistance: structure and function of an unique gastrointestinal epithelial barrier. J Lab Clin Med 1994;123:653-9.

8 Flemström G, Isenberg Jl. Gastroduodenal mucosal alkaline secretion and mucosal protection. News Physiol Sci 2001;16:23-8

9 Orlando RC, Lacy ER, Tobey NA, et al. Barriers to paracellular permeability in rabbit esophageal epithelium. Gastroenterology 1992:102:910-23.

10 McKie LD, Dunkin BJ, Pennanen MF, et al. Esophageal mucosal blood flow: a central role for calcitonin gene-related peptide. Surgery 1994; 116:409-17.

11 Brown CM, Rees WD. Review article: factors protecting the oesophagus against acid-mediated injury. Aliment Pharmacol Ther 1995;9:251-62.

12 Sarosiek J, Yu Z, Namiot Z, et al. Impact of acid and pepsin on human esophageal prostaglandins. Am J Gastroenterol 1994;89:588-94.

13 Stolte M, Vieth M, Schmitz JM, et al. Effects of long-term treatment with proton pump inhibitors in gastro-oesophageal reflux disease on the histological findings in the lower oesophagus. Scand J Gastroenterol 35 : 1125-30.

14 Tobey NA, Orlando RC. Mechanisms of acid injury to rabbit esophageal epithelium. Role of basolateral cell membrane acidification. Gastroenterology1991;101:1220-8

15 Tobey NA, Powell DW, Schreiner VJ, et al. Serosal bicarbonate protects against acid injury to rabbit esophagus. Gastroenterology 1989:96:1466-77.

16 Akiba Y, Kaunitz JD. Regulation of intracellular $\mathrm{pH}$ and blood flow in rat duodenal epithelium in vivo. Am J Physiol Gastrointest Liver Physiol 1999:276:G293-302.

17 Mordon S, Devoisselle JM, Maunoury V. In vivo $\mathrm{pH}$ measurement and imaging of tumor tissue using a $\mathrm{pH}$-sensitive fluorescent probe (5,6-carboxyfluorescein): instrumental and experimental studies. Photochem Photobiol 1994;60:274-9.

18 Chu S, Tanaka S, Kaunitz JD, et al. Dynamic regulation of gastric surface $\mathrm{pH}$ by luminal $\mathrm{pH}$. J Clin Invest 1999; 103:605-12.

19 Chu SY, Brownell WE, Montrose MH. Quantitative confocal imaging along the crypt- to-surface axis of colonic crypts. Am J Physiol Cell Physiol 1995;269: C1557-64

20 Rowe WA, Lesho $\mathrm{M}$, Montrose $\mathrm{MH}$. Polarized $\mathrm{Na}^{+} / \mathrm{H}^{+}$exchange function is pliable in response to transepithelial gradients of propionate. Proc Natl Acad Sci U S A 1994:91:6166-70.

21 Chu S, Montrose MH. Extracellular pH regulation in microdomains of colonic crypts - effects of short-chain fatty acids. Proc Natl Acad Sci U S A 1995;92:3303-7

22 Kaneko K, Guth PH, Kaunitz JD. In vivo measurement of rat gastric surface cell intracellular pH. Am J Physiol Gastrointest Liver Physiol 1991;261:G548-52.

23 Martin GR, Jain RK. Noninvasive measurements of interstitial pH profiles in normal and neoplastic tissue fluorescence ratio imaging microscopy. Cancer Res 1994;54:5670-4.

24 Aharinejad S, Lametschwandtner A, Franz P, et al. The vascularization of the digestive tract studied by scanning electron microscopy with special emphasis on the teeth, esophagus, stomach, small and large intestine, pancreas, and liver Scanning Microsc 1991:5:81 1-49.

25 Kaunitz JD, Nishizaki Y, Kaneko K, et al. Effect of orogastric nicotine on rat gastric mucosal gel thickness, surface cell viability, and intracellular $\mathrm{pH}$. J Pharmacol Exp Ther 1993;265:948-54.

26 Akiba Y, Guth PH, Engel E, et al. Dynamic regulation of mucus gel thickness in rat duodenum. Am J Physiol Gastrointest Liver Physiol 2000;279:G437-47.

27 Akiba Y, Guth PH, Engel E, et al. Acid-sensing pathways of rat duodenum. Am J Physiol Gastrointest Liver Physiol 1999;277:G268-74.

28 Gonda T, Maouyo D, Rees SE, et al. Regulation of intracellular $\mathrm{pH}$ gradients by identified $\mathrm{Na} / \mathrm{H}$ exchanger isoforms and a short-chain fatty acid. Am J Physiol Gastrointest Liver Physiol 1999;276: G259-70.
29 Northway MG, Eastwood GL, Libshitz HI, et al. Antiinflammatory agents protect opossum esophagus during radiotherapy. Dig Dis Sci 1982:27:923-8.

30 Northway MG, Libshitz HI, Osborne BM, et al. Radiation esophagitis in the opossum: radioprotection with indomethacin. Gastroenterology 1980;78:883-92.

31 Cryer B. Mucosal defense and repair. Role of prostaglandins in the stomach and duodenum. Gastroentreral Clin North Am 2001;30:877-94

32 Holzer P. Neural emergency system in the stomach. Gastroenterology 1998;114:823-39.

33 Abdel-Salam OM, Czimmer J, Debreceni A, et al. Gastric mucosal integrity: gastric mucosal blood flow and microcirculation. An overview. J Physiol Paris 2001;95:105-27.

34 Ghillebert G, Demeyere AM, Janssens J, et al. How well can quantitative 24-hour intraesophageal $\mathrm{pH}$ monitoring distinguish various degrees of reflux disease? Dig Dis Sci 1995:40:1317-24.

35 Khalbuss W, Marousis CG, Subramanyam M, et al. Effect of $\mathrm{HCl}$ on transmembrane potentials and intracellular $\mathrm{pH}$ in rabbit esophageal epithelium. Gastroenterology 1995;108:662-72

36 Orlando RC, Powell DW, Carney CN. Pathophysiology of acute acid injury in rabbit esophageal epithelium. J Clin Invest 1981;68:286-93.

37 Orlando RC. Esophageal epithelial defenses against acid injury. Am Gastroenterol 1994;89:S48-52.

38 Akiba Y, Furukawa O, Guth $\mathrm{PH}$, et al. Sensory pathways and cyclooxygenase regulate mucus gel thickness in rat duodenum. Am J Physiol Gastrointest Liver Physiol 2001;280:G470-4.

39 Shi G, Tatum RP, Joehl R, et al. Esophageal sensitivity and symptom perception in gastroesophageal reflux disease. Curr Gastroenterol Rep $1999 ; 1: 214-19$

40 Dutsch $\mathrm{M}$, Eichhorn U, Worl J, et al. Vagal and spinal afferent innervation of the rat esophagus: a combined retrograde tracing and immunocytochemical study with special emphasis on calcium-binding proteins. J Comp Neurol 1998;398:289-307

41 Williams SE, Turnberg LA. Retardation of acid diffusion by pig gastric mucosa: a potential role in mucosal protection. Gastroenterology1980;79:299-304.

42 Teeter BC, Helm JF, Linehan J, et al. Computerized model of the mechanisms governing esophageal acid clearance. Dig Dis Sci 1982:27:664

43 Dixon J, Strugala V, Griffin SM, et al. Esophageal mucin: an adherent mucus gel barrier is absent in the normal esophagus but present in columnar-lined Barrett's esophagus. Am J Gastroenterol 2001;96:2575-83

44 Kinoshita $M$, Kume E, Igarashi $S$, et al. Role of salivary mucin in the protection of rat esophageal mucosa from acid and pepsin-induced injury. Am J Physiol Gastrointest Liver Physiol 1999;277:G796-800.

45 Nemes Z, Steinert PM. Bricks and mortar of the epidermal barrier. Exp Mol Med 1999;31:5-19.

46 Hamilton BH, Orlando RC. In vivo alkaline secretion by mammalian esophagus. Gastroenterology1989;97:640-8.

47 Poorkhalkali N, Jacobson I, Helander HF. Lectin histochemistry of the esophagus in several mammalian species. Anat Embryol (Berl) 1999.200:541-9

48 Gipson IK, Spurr-Michaud SJ, Tisdale AS, et al. Stratified squamous epithelia produce mucin-like glycoproteins. Tissue Cell 1995;27:397-404

49 Hollwarth ME, Smith M, Kvietys PR, et al. Esophageal blood flow in the cat. Normal distribution and effects of acid perfusion. Gastroenterology 1986:90:622-7.

50 Johnson LF, Harmon JW. Experimental esophagitis in a rabbit model. Clinical relevance. J Clin Gastroenterol 1986;8(suppl 1):26-44.

51 Harmon JW, Woods M, Gurll NJ. Different mechanisms of hydrogen ion removal in stomach and duodenum. Am J Physiol 1978;235:E692-98.

52 Duda G, Huesken JE, Harmon JW. Effects of prostaglandins on esophageal blood flow. J Surg Res 1988;44:371-81.

53 Bass BL, Trad KS, Harmon JW, et al. Capsaicin-sensitive nerves mediate esophageal mucosal protection. Surgery1991:110:419-25.

54 McKie LD, Bass BL, Dunkin BJ, et al. Nitric oxide regulates basal but not capsaicin-, CGRP-, or bile salt-stimulated rabbit esophageal mucosal blood flow. Ann Surg 1995;222:186-92.

55 Sababi $M$, Nylander $O$. Comparative study of the effects of nitric oxide synthase and cyclo-oxygenase inhibition on duodenal functions in rats anaesthetized with inactin, urethane or alpha-chloralose. Acta Physiol Scand 1996; 158:45-52

56 Sugamoto S, Kawauch S, Furukawa $O$, et al. Role of endogenous nitric oxide and prostaglandin in duodenal bicarbonate response induced by mucosal acidification in rats. Dig Dis Sci 2001;46:1208-16.

57 Morgan G. Deleterious effects of prostaglandin $E_{2}$ in reflux oesophagitis. Med Hypotheses 1996;46:42-4. 